\title{
Características y consecuencias de las agresiones sexuales en adolescentes consultantes en un centro de salud sexual y reproductiva
}

\author{
Electra González A. ${ }^{1 a}$, Adela Montero V. ${ }^{1}$, Vania Martínez N. ${ }^{1}$, Carolina Leyton M. ${ }^{1 b}$, \\ Carolina Luttges $D .{ }^{1 b}$, Temístocles Molina G. $1 \mathrm{c}$ \\ ${ }^{1}$ Centro de Medicina Reproductiva y Desarrollo Integral del Adolescente (CEMERA), Facultad de Medicina, Universidad \\ de Chile.
}

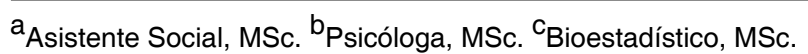

\section{RESUMEN}

Antecedentes: La agresión sexual es un evento traumático y doloroso que afecta de manera transversal, a las víctimas de ambos sexos, de todos los niveles socioeconómicos con graves consecuencias para la salud física, psicológica y social para quienes la sufren. Objetivo: Determinar las características de las agresiones sexuales y sus consecuencias en adolescentes consultantes en un centro de atención en Salud Sexual y Reproductiva. Método: Estudio analítico de corte transversal de 573 adolescentes atendidas en un centro de medicina reproductiva (CEMERA) en el período 2003-2010 y que reportaron antecedente de abuso sexual. Se estudiaron tanto las características como las consecuencias del abuso, datos que se obtuvieron desde las fichas clínicas. Resultados: En el $86,5 \%$ de los casos se reportó abuso sexual y en un $13,5 \%$ violación. El $48,5 \%$ de los agresores son familiares. La edad más vulnerable fue entre 5-9 años con un $41,2 \%$. En el $39,2 \%$ el abuso sexual fue reiterado. En un $43,4 \%$ el lugar fue el propio hogar de la víctima. Un $70 \%$ reveló y de estos el $45,9 \%$ lo hizo en forma tardía. Sólo en un $19 \%$ se hizo la denuncia legal. De las adolescentes que iniciaron actividad sexual voluntaria (83,3\%), un $35,4 \%$ tenía menos de 15 años. Un $11,8 \%$ reportó 3 y más parejas sexuales. Conclusión: El antecedente de abuso sexual debe ser considerado en la atención de adolescentes que consultan por salud sexual y reproductiva. El equipo de salud debe estar preparado para atender sus múltiples necesidades.

\section{PALABRAS CLAVE: Agresiones sexuales, adolescentes, consecuencias}

\section{SUMMARY}

Background: Sexual assault is a traumatic and painful event, which affects victims of both genders, across all socioeconomic levels, with serious consequences for physical, psychological, and social health. Objective: Determine the characteristics of sexual assault and its consequences in adolescents at a sexual and reproductive health center. Method: Analytic and cross-sectional study of 573 adolescents seen at a reproductive medicine center (CEMERA) in the period 2003-2010 and who reported history of sexual abuse. Patient's clinical records were reviewed to study the characteristics as well as the consequences of sexual abuse. Results: The abuse was reported as sexual abuse in $86.5 \%$ of the cases and as rape in $13.5 \%$. In $48.5 \%$ of the cases, the aggressors were relatives of the victims. The most vulnerable group is between 5-9 of age, representing $41.2 \%$ of the cases. For $39.2 \%$ of the cases, the sexual abuse was reiterative, and in $43.4 \%$, the abuse took 
place on the victim's home. $70 \%$ of the victims told someone about the abuse; $45.9 \%$ of those revealed their abuse in a delay manner. Only $19 \%$ of victims filed a legal complaint. Of the adolescents who started voluntary sexual activity (83.3 \%) a $35.4 \%$ was under de age of 15 , and $11,8 \%$ reported having 3 or more sexual partners. Conclusion: A history of sexual abuse must be considered in the attention of adolescents who consult for reproductive and sexual health. The health team must be prepared to address their multiple needs.

\section{KEY WORDS: Sexual assaults, adolescents, consequences}

\section{INTRODUCCIÓN}

La agresión sexual es un evento traumático y doloroso que afecta, de manera transversal, a las víctimas de ambos sexos, y de todos los niveles socioeconómicos. Sin embargo, la mayoría de los estudios muestran que las víctimas de abuso sexual son más frecuentemente mujeres y los agresores generalmente son hombres. Por otro lado, los casos que más se reportan corresponden a mujeres de sectores socioeconómicos más pobres. Que la violencia sexual sea más frecuente en las mujeres puede ser explicado por diferencias de género a nivel cultural en la que la mujer, no importando su edad, es considerada como propiedad del varón. A pesar de que una mujer puede ser atacada sexualmente en cualquier etapa de su vida, la etapa más vulnerable es la niñez y la adolescencia (1-4).

En Chile durante el año 2010 se reportaron 6.956 denuncias por agresiones sexuales, de éstas 5.444 afectaron a mujeres y 1.512 a hombres. En la Región Metropolitana es donde mayormente se concentró el fenómeno con 3.452 denuncias, siendo 1.139 por violación y 2.311 por abuso sexual. Las cifras nacionales nos indican mayor cantidad de víctimas mujeres que hombres; las edades más vulnerables son entre 5 y 14 años; los victimarios conocidos no familiares representan el $51 \%$ de los casos; los familiares corresponden al $31 \%$ y los desconocidos al $18 \%$ (5).

La mayoría de los traumas causados por las agresiones sexuales son psicológicos, con importantes consecuencias a largo plazo, que afectarán su vida social, familiar y sexual futura.

En el área de salud mental, estudios realizados tanto en el área clínica como en comunidades, han reportado que la depresión es uno de los síntomas más reportados por mujeres con historia de abuso sexual. Otros efectos que se reportan son: comportamientos autodestructivos, auto agresiones, sentimientos suicidas, síntomas de ansiedad y estrés, pesadillas, trastornos del sueño, auto concepto y auto imagen negativa, sentimientos de aislamiento y estigmatización (6-8).
En el área de las relaciones interpersonales, se presentan serios problemas en la relaciones con personas de ambos sexos, como también pueden aparecer sentimientos de hostilidad hacia sus padres porque no los protegieron del abuso. Se ha observado también relaciones conflictivas con sus propios hijos. Como madres, pueden no ser capaces de darse cuenta de si su hijo o hija está siendo víctima de abuso sexual y tienden a mantener distancia emocional y física con ellos, lo cual puede constituir a su vez en un factor de riesgo para el abuso de estos niños; o por el contrario, mantener una desconfianza extrema de toda persona que se acerque a su hijo o hija. Tienen dificultades para confiar en las personas, con reacciones de temor, hostilidad y sentimientos de amenaza $(8,9)$.

Por otro lado, las mujeres víctimas de incesto presentan mayores dificultades en sus relaciones cercanas, como temor a sus esposos o parejas. Finalmente otro impacto es la vulnerabilidad de estas mujeres para ser víctimas nuevamente de abuso sexual (10-12).

En el área de la sexualidad, la mayoría de los estudios muestran serias consecuencias, particularmente, las víctimas de incesto muestran mayores niveles de ansiedad, en la vida sexual, culpa sexual y una gran insatisfacción en sus relaciones sexuales cuando se comparan con mujeres sin antecedente de abuso sexual infantil. Inhabilidad para relajarse y disfrutar la actividad sexual, abstinencia sexual y otras disfunciones sexuales están presentes en estas mujeres. Ellas experimentan menos orgasmos, y obtienen una baja satisfacción de sus parejas sexuales, se sienten menos satisfechas de la calidad de las relaciones que establecen con los varones, y reportan más parejas sexuales comparadas con aquellas que no sufrieron esta experiencia. También se ha observado un mayor nivel de comportamientos sexuales riesgosos, probablemente con el propósito de obtener afecto y atención (13-18).

En el área de las funciones sociales, la literatura muestra una relación entre abuso sexual en la niñez o adolescencia e involucramiento en comercio sexual y/o prostitución en la adultez, como 
también, una fuerte asociación con otras conductas de riesgo como abuso de alcohol y drogas (19-23). Finalmente las infecciones de transmisión sexual, entre ellas el VIH-SIDA, y el embarazo producto de violación son otras de las graves consecuencias reportadas del abuso sexual (24-33).

La legislación penal chilena define violación como: "el que accede carnalmente, por vía vaginal, anal o bucal, a una persona mayor de 14 años en algunos de los casos siguientes, cuando se use de fuerza o intimidación, cuando la víctima se halle privada de sentido, o cuando se aprovecha su incapacidad para oponer resistencia, cuando se abusa de la enajenación o trastorno mental de la víctima" y abuso sexual "el que abusivamente realizare una acción sexual distinta del acceso carnal con una persona menor de 14 años. Cualquier acto de significación sexual y de relevancia realizado mediante contacto corporal con la víctima, o que haya afectado los genitales, el ano o la boca de la víctima, aún cuando no hubiere contacto corporal con ella" (34).

El objetivo del presente estudio fue determinar las características de las agresiones sexuales y sus consecuencias en adolescentes consultantes en un Centro de Atención en Salud Sexual y Reproductiva.

\section{PACIENTES Y MÉTODO}

Estudio analítico de corte transversal. Los datos se obtuvieron desde las fichas clínicas de los adolescentes que ingresaron al Centro de Medicina Reproductiva y Desarrollo Integral del Adolescente (CEMERA) desde enero 2003 hasta diciembre 2010. CEMERA es un centro universitario de salud sexual y reproductiva que provee atención integral y confidencial para adolescentes de nivel socioeconómico medio y bajo. Las adolescentes consultan predominantemente por servicios anticonceptivos siendo evaluadas por un equipo multiprofesional compuesto por ginecólogos, matrona, psiquiatra, psicólogas y asistente social, quienes se han especializados en la atención de adolescentes. A su ingreso los y las adolescentes reciben información sobre la modalidad de atención y características del Centro, solicitando explícitamente autorización para poder utilizar los datos que se consignaran en la ficha clínica para fines de investigación con resguardo de los aspectos éticos. Lo anterior se traduce en la posterior firma de un consentimiento informado elaborado para estos fines.

Durante el período de estudio, 3.497 adolescentes consultaron en CEMERA. De estos, 746 reportaron antecedente de agresión sexual $(21,6 \%)$. Para este estudio sólo se consideró a las mujeres lo que corresponde a 573 casos.

Las variables analizadas en este estudio fueron aquellas relacionadas con las características de la agresión sexual como: tipo de agresión sexual de acuerdo a lo que contempla nuestra legislación (violación o abuso sexual), duración de la agresión sexual (única o reiterada), edad de la víctima a la agresión, relación víctima - victimario, edad del agresor, lugar de la agresión, revelación de la agresión sexual (sí-no), tiempo de la revelación (inmediata o tardía), tiempo transcurrido entre el abuso y la revelación (en años), a quién reveló, medidas tomadas por la persona a quien reveló la información, realización de denuncia legal, resultado de la denuncia. También las relacionadas con las conductas de riesgo como consecuencias asociadas a la agresión sexual: actividad sexual voluntaria, inicio de la actividad sexual voluntaria antes de los 15 años, número de parejas sexuales, consumo de alcohol, consumo de drogas. Esta investigación contó con la aprobación del Comité de Ética en Seres Humanos de la Facultad de Medicina de la Universidad de Chile.

Análisis Estadístico. Los resultados para las variables (discretas y continuas) medidas en escala numérica, fueron expresados como valores promedios y porcentajes para las medidas en escala nominal. Se realizaron análisis uni y bivariado aplicándose las pruebas estadísticas: test Chi2, test diferencia de medias, test de Wilcoxon, ANOVA, Kruskal-Wallis. El análisis bivariado analizó además las variables asociadas a la agresión sexual con la variable resultado, consecuencias o comportamiento de riesgo (el inicio voluntario de la actividad sexual, edad del inicio de la actividad sexual, número de parejas sexuales, consumo de alcohol y drogas).

\section{RESULTADOS}

El promedio de edad de las adolescentes fue de 15,8 años (rango: 10-19 años). El promedio de edad a la agresión sexual fue de 10,1 años (rango: 2-18 años).

Como formas de agresión sexual en el $86,5 \%$ de los casos se reportó abuso sexual y en un 13,5\% violación. El $48,5 \%$ los agresores son familiares, el $34,9 \%$ conocidos y en un $16,5 \%$ corresponde a desconocido. En un 34,2\% los agresores tenían 19 años y menos. En el $41,2 \%$ la edad de la víctima en el momento de la primera agresión sexual fue entre 5 y 9 años, un $41 \%$ entre 10 y 14 años. En el $60,8 \%$ el abuso sexual fue único y en el $39,2 \%$ reiterado. En un $43,4 \%$ el lugar fue el propio hogar de la víctima. Un $70 \%$ reveló la agresión sexual y 
de éstos el $45,9 \%$ lo hizo en forma tardía. Se reporta a la madre en un $59 \%$, a familiar en $19,6 \%$. Al revelarse se tomó alguna medida en dos tercios de las agresiones. Sólo en un 19\% se hizo la denuncia legal (Tabla I).

Tabla I

VARIABLES RELACIONADAS CON LA AGRESIÓN SEXUAL

\begin{tabular}{|c|c|c|c|}
\hline Variable & Categorías & $\mathrm{n}$ & $\%$ \\
\hline Edad a la entrevista (años) & $\begin{array}{l}10-14 \\
15-16 \\
17-19\end{array}$ & $\begin{array}{r}85 \\
291 \\
195\end{array}$ & $\begin{array}{l}14,9 \\
51,0 \\
34,1\end{array}$ \\
\hline Tipo de agresión sexual & $\begin{array}{l}\text { Abuso sexual } \\
\text { Violación }\end{array}$ & $\begin{array}{r}467 \\
73\end{array}$ & $\begin{array}{l}86,5 \\
13,5\end{array}$ \\
\hline Duración de la agresión & $\begin{array}{l}\text { Única } \\
\text { Reiterada }\end{array}$ & $\begin{array}{l}290 \\
187\end{array}$ & $\begin{array}{l}60,8 \\
39,2\end{array}$ \\
\hline Edad a la agresión (años) & $\begin{array}{l}2-4 \\
5-9 \\
10-14 \\
15-18\end{array}$ & $\begin{array}{r}21 \\
215 \\
214 \\
72\end{array}$ & $\begin{array}{r}4,0 \\
41,2 \\
41,0 \\
13,8\end{array}$ \\
\hline Relación víctima-victimario & $\begin{array}{l}\text { Padre } \\
\text { Padrastro } \\
\text { Hermano } \\
\text { Abuelo } \\
\text { Otro familiar } \\
\text { Conocido } \\
\text { Desconocido }\end{array}$ & $\begin{array}{r}24 \\
44 \\
11 \\
22 \\
102 \\
146 \\
69\end{array}$ & $\begin{array}{r}5,7 \\
10,5 \\
2,6 \\
5,3 \\
24,4 \\
34,9 \\
16,5\end{array}$ \\
\hline Edad del agresor (años) & $\begin{array}{l}15 \text { y menos } \\
16-19 \\
20-90\end{array}$ & $\begin{array}{r}48 \\
60 \\
217\end{array}$ & $\begin{array}{l}14,8 \\
14,4 \\
66,8\end{array}$ \\
\hline Lugar de ocurrencia de la agresión & $\begin{array}{l}\text { Domicilio víctima } \\
\text { Domicilio víctima-victimario } \\
\text { Domicilio victimario } \\
\text { Domicilio familiar } \\
\text { Domicilio conocido } \\
\text { Colegio } \\
\text { Vía pública }\end{array}$ & $\begin{array}{r}62 \\
43 \\
38 \\
20 \\
17 \\
8 \\
54\end{array}$ & $\begin{array}{r}25,6 \\
17,8 \\
15,7 \\
8,3 \\
7,0 \\
3,3 \\
22,3\end{array}$ \\
\hline Revelación & $\begin{array}{l}\mathrm{Si} \\
\text { No }\end{array}$ & $\begin{array}{l}377 \\
161\end{array}$ & $\begin{array}{l}70,0 \\
30,0\end{array}$ \\
\hline A quien reveló & $\begin{array}{l}\text { Madre o padre } \\
\text { Familiar } \\
\text { Conocido } \\
\text { Desconocido }\end{array}$ & $\begin{array}{r}220 \\
73 \\
71 \\
9\end{array}$ & $\begin{array}{r}59,0 \\
19,6 \\
19,0 \\
2,4\end{array}$ \\
\hline $\begin{array}{l}\text { Medidas que tomó esa persona a } \\
\text { quien reveló }\end{array}$ & $\begin{array}{l}\text { Ninguna } \\
\text { Enfrentó al agresor } \\
\text { Lo denunció } \\
\text { Tomo precauciones } \\
\text { No le creyó }\end{array}$ & $\begin{array}{l}66 \\
80 \\
59 \\
80 \\
25\end{array}$ & $\begin{array}{r}21,3 \\
25,8 \\
19,0 \\
25,8 \\
8,0\end{array}$ \\
\hline Tiempo que demoró en revelar & $\begin{array}{l}\text { Inmediata } \\
\text { Tardía }\end{array}$ & $\begin{array}{r}113 \\
96\end{array}$ & $\begin{array}{l}54,1 \\
45,9\end{array}$ \\
\hline Denunció a la justicia & $\begin{array}{l}\mathrm{Si} \\
\text { No }\end{array}$ & $\begin{array}{r}59 \\
251\end{array}$ & $\begin{array}{l}19,0 \\
81,0\end{array}$ \\
\hline
\end{tabular}


Un $83,3 \%$ había iniciado actividad sexual voluntaria, el $35,4 \%$ inició antes de los 15 años, un $65,8 \%$ reportó una pareja sexual. Un $79,3 \%$ reportó consumo de alcohol, casi la mitad había consumía variados tipos de bebidas alcohólicas, y la frecuencia más reportada fue los fines de semana. Un $43,7 \%$ reportó haber consumido drogas, de ellas la más frecuente fue la marihuana con un 90,9\% (Tabla II).

No se encontró diferencia cuando se analizó tipo de agresión o si reveló o no, con inicio de actividad sexual voluntaria, ni con edad de inicio de la actividad sexual voluntaria. Las adolescentes que sufrieron violación tienen más parejas sexuales cuando se comparan con las que sufrieron abusos sexuales ( 3 y más parejas sexuales: $23,1 \%$ vs 10,6\%; $p=0,02$ ) (Tabla III). Hubo asociación entre menor edad a la agresión y no revelación $(p=0,05)$ $y$ consumo de drogas $(p=0,03)$. Mientras que menor edad a la agresión sexual y no revelación se asoció a consumo de drogas (Tabla IV).

La violación se cometió con mayor frecuencia en la casa de un familiar cuando se compara con el abuso sexual. Mayor número de parejas sexuales se observó en las adolescentes que sufrieron violación. El promedio de edad de los agresores que cometieron abuso sexual es mayor que aquellos que cometieron violación (Tabla $\mathrm{V}$ ).

La revelación del abuso sexual se asoció significativamente a agresor familiar ( $p=0,00)$, a no consumo de drogas $(p=0,03)$ y mayor edad del agresor $(p=0,00)($ Tabla VI).

Tabla II

VARIABLES CONDUCTUALES DE RIESGO QUE PRESENTARON LAS ADOLESCENTES DEL ESTUDIO

\begin{tabular}{|c|c|c|c|}
\hline Variable & Categorías & $\mathrm{n}$ & $\%$ \\
\hline Inicio de actividad sexual voluntaria & $\begin{array}{l}\mathrm{Si} \\
\mathrm{No}\end{array}$ & $\begin{array}{r}470 \\
94\end{array}$ & $\begin{array}{l}83,3 \\
16,7\end{array}$ \\
\hline Edad inicio actividad sexual voluntaria (años) & $\begin{array}{l}9-14 \\
15-18\end{array}$ & $\begin{array}{l}166 \\
303\end{array}$ & $\begin{array}{l}35,4 \\
64,6\end{array}$ \\
\hline Número de parejas sexuales & $\begin{array}{l}1 \\
2 \\
\geq 3\end{array}$ & $\begin{array}{r}306 \\
104 \\
55\end{array}$ & $\begin{array}{l}65,8 \\
22,4 \\
11,8\end{array}$ \\
\hline Consumo de alcohol & $\begin{array}{l}\mathrm{Si} \\
\mathrm{No}\end{array}$ & $\begin{array}{l}449 \\
117\end{array}$ & $\begin{array}{l}79,3 \\
20,7\end{array}$ \\
\hline Tipo de alcohol que consume & $\begin{array}{l}\text { Cerveza } \\
\text { Vino } \\
\text { Pisco } \\
\text { Ron } \\
\text { Varios }\end{array}$ & $\begin{array}{r}134 \\
29 \\
28 \\
21 \\
212\end{array}$ & $\begin{array}{r}31,6 \\
6,8 \\
6,6 \\
4,9 \\
50,0\end{array}$ \\
\hline Frecuencia del consumo & $\begin{array}{l}\text { Fin de semana } \\
1 \text { a } 2 \text { veces al mes } \\
\text { Ocasional } \\
\text { Experimental }\end{array}$ & $\begin{array}{r}203 \\
115 \\
55 \\
30\end{array}$ & $\begin{array}{r}50,4 \\
28,5 \\
13,7 \\
7,4\end{array}$ \\
\hline Consumo de droga & $\begin{array}{l}\mathrm{Si} \\
\mathrm{No}\end{array}$ & $\begin{array}{l}246 \\
317\end{array}$ & $\begin{array}{l}43,7 \\
56,3\end{array}$ \\
\hline Tipo de droga que consume & $\begin{array}{l}\text { Marihuana } \\
\text { Otra }\end{array}$ & $\begin{array}{r}220 \\
22\end{array}$ & $\begin{array}{r}90,9 \\
9,1\end{array}$ \\
\hline Frecuencia del consumo de droga & $\begin{array}{l}\text { Diaria } \\
\text { Semanal } \\
\text { Mensual } \\
\text { Ocasional } \\
\text { Experimental }\end{array}$ & $\begin{array}{l}27 \\
47 \\
26 \\
52 \\
79\end{array}$ & $\begin{array}{l}11,7 \\
20,5 \\
11,3 \\
22,5 \\
34,2\end{array}$ \\
\hline
\end{tabular}


Tabla III

VARIABLES RELACIONADAS CON LA AGRESIÓN SEXUAL SEGÚN NÚMERO DE PAREJAS SEXUALES

\begin{tabular}{|c|c|c|c|c|c|c|c|c|}
\hline \multirow{3}{*}{ Variables } & \multicolumn{6}{|c|}{ Número de parejas sexuales } & \multirow{3}{*}{ Total } & \multirow{3}{*}{ Valor $\mathrm{p}$} \\
\hline & \multicolumn{2}{|c|}{1} & \multicolumn{2}{|c|}{2} & \multicolumn{2}{|c|}{$\geq 3$} & & \\
\hline & $\mathrm{n}$ & $\%$ & $\mathrm{n}$ & $\%$ & $\mathrm{n}$ & $\%$ & & \\
\hline \multicolumn{9}{|l|}{ Tipo de agresión } \\
\hline Abuso sexual & 260 & 67,2 & 86 & 22,2 & 41 & 10,6 & 387 & \\
\hline Violación & 27 & 51,9 & 13 & 25,0 & 12 & 23,1 & 52 & 0,02 \\
\hline \multicolumn{9}{|l|}{ Duración de la agresión } \\
\hline Único & 150 & 64,9 & 57 & 24,7 & 24 & 10,4 & 231 & 0,44 \\
\hline Reiterado & 106 & 68,4 & 30 & 19,4 & 19 & 12,3 & 155 & \\
\hline \multicolumn{9}{|l|}{ Relación víctima-victimario } \\
\hline Familiar & 165 & 66,0 & 54 & 21,6 & 31 & 12,4 & 250 & 0,85 \\
\hline No familiar & 132 & 65,0 & 48 & 23,6 & 23 & 11,3 & 203 & \\
\hline \multicolumn{9}{|l|}{ Lugar de ocurrencia } \\
\hline Casa víctima & 57 & 67,9 & 19 & 22,6 & 8 & 9,5 & 84 & 0,06 \\
\hline Casa de familiar o conocido & 22 & 68,8 & 3 & 9,4 & 7 & 21,9 & 32 & \\
\hline Otro lugar & 51 & 65,4 & 22 & 28,2 & 5 & 6,4 & 78 & \\
\hline \multicolumn{9}{|l|}{ Revelación } \\
\hline Sí & 208 & 66,9 & 69 & 22,2 & 34 & 10,9 & 311 & 0,49 \\
\hline No & 80 & 62,9 & 28 & 22,1 & 19 & 14,9 & 127 & \\
\hline \multicolumn{9}{|l|}{ Tiempo que demoró en revelar } \\
\hline Inmediata & 59 & 62,8 & 28 & 29,8 & 7 & 7,5 & 94 & 0,07 \\
\hline Tardía & 49 & 62,1 & 16 & 20,3 & 14 & 17,7 & 79 & \\
\hline \multicolumn{9}{|l|}{ Edad a la agresión } \\
\hline (promedio, años) & 10,2 & & 9,7 & & 9,9 & & & 0,44 \\
\hline \multicolumn{9}{|l|}{ Edad agresor } \\
\hline (promedio, años) & 30,1 & & 30,0 & & 29,3 & & & 0,78 \\
\hline
\end{tabular}

\section{DISCUSIÓN}

Concordante con los datos reportados por el Servicio Medico Legal (SML) (5) el presente estudio encontró que la edad más vulnerable para la agresión fue entre 5 y 14 años, y que los abusos sexuales fueron más frecuentes que la violación, pero los agresores familiares representan la mayoría de los victimarios a diferencia de los datos del SML, que reportó a los conocidos no familiares con mayor frecuencia. Este antecedente es muy relevante porque además indica que los espacios donde se realizó el delito es el espacio privado o familiar del hogar, ya sea de la víctima o del victimario; el $67 \%$ ocurrió en este espacio, en donde además, el agresor tiene un lazo vinculante consanguíneo, directo o no, con la víctima. Esta circunstancia hace más difícil la revelación a algún familiar o persona de confianza y cuando ésta ocurre es generalmente tardía. La denuncia a la justicia tiende a ser más infrecuente, en nuestro estudio alcanzó el $19 \%$, esto puede ser explicado por el temor a reconocer en lo público este tipo de prácticas o a la desconfianza que se tiene en el sistema judicial o por estar la víctima bajo amenaza del agresor.

Los análisis de asociación entre las características de la agresión sexual y las conductas de riesgo, muestran que las adolescentes que sufrieron violación tienen mas parejas sexuales, que la menor edad de ocurrencia de la agresión se asoció a consumo de alcohol y que la revelación se asoció a que el tipo de agresor era familiar y no al consumo de drogas.

Considerando el impacto del abuso sexual en la 


\section{Tabla IV}

\section{VARIABLES RELACIONADAS CON LA AGRESIÓN SEXUAL SEGÚN CONSUMO DE DROGAS}

\begin{tabular}{|c|c|c|c|c|c|c|}
\hline \multirow{3}{*}{ Variable } & \multicolumn{4}{|c|}{ Consumo de drogas } & \multirow{3}{*}{ Total } & \multirow{3}{*}{ Valor $p$} \\
\hline & \multicolumn{2}{|c|}{$\mathrm{Si}$} & \multicolumn{2}{|c|}{ No } & & \\
\hline & $\mathrm{n}$ & $\%$ & $\mathrm{n}$ & $\%$ & & \\
\hline \multicolumn{7}{|l|}{ Tipo de agresión } \\
\hline Abuso sexual & 199 & 43,1 & 263 & 56,9 & 462 & 0,39 \\
\hline Violación & 35 & 49,3 & 36 & 50,7 & 71 & \\
\hline \multicolumn{7}{|l|}{ Duración de la agresión } \\
\hline Único & 130 & 45,3 & 157 & 54,7 & 287 & 0,22 \\
\hline Reiterado & 72 & 39,1 & 112 & 60,9 & 184 & \\
\hline \multicolumn{7}{|l|}{ Relación víctima-victimario } \\
\hline Familiar & 136 & 46,1 & 159 & 53,9 & 295 & 0,32 \\
\hline No Familiar & 105 & 41,5 & 148 & 58,5 & 253 & \\
\hline \multicolumn{7}{|l|}{ Lugar de ocurrencia } \\
\hline Casa víctima & 45 & 43,3 & 59 & 56,7 & 104 & \\
\hline Casa de familiar o conocido & 12 & 32,4 & 25 & 67,6 & 37 & 0,34 \\
\hline Otro lugar & 34 & 34,7 & 64 & 65,3 & 98 & \\
\hline \multicolumn{7}{|l|}{ Revelación } \\
\hline Sí & 152 & 40,8 & 221 & 59,3 & 373 & \\
\hline No & 81 & 51,3 & 77 & 48,7 & 158 & 0,03 \\
\hline \multicolumn{7}{|l|}{ Tiempo que demoró en revelar } \\
\hline Inmediata & 44 & 38,9 & 69 & 61,1 & 113 & 0,32 \\
\hline Tardía & 44 & 46,8 & 50 & 53,2 & 94 & \\
\hline \multicolumn{7}{|l|}{ Edad a la agresión } \\
\hline (promedio, años) & 9,8 & & 10,4 & & & 0,05 \\
\hline \multicolumn{7}{|l|}{ Edad del agresor } \\
\hline (promedio, años) & 30,5 & & 30,4 & & & 0,82 \\
\hline
\end{tabular}

salud de las víctimas, es necesario conocer el antecedente de esta forma de violencia por el equipo de salud que atiende adolescentes con el objetivo de diseñar estrategias orientadas a satisfacer las múltiples necesidades en los aspectos biopsicosociales y legales, así como acciones destinadas a la prevención de las agresiones sexuales. Los resultados del estudio pueden ser una contribución significativa al conocimiento de esta temática como al entrenamiento de profesionales, que de una u otra manera, tienen la responsabilidad de atender a adolescentes que presentan una historia de abuso sexual, mejorando la calidad de la atención con promoción de medidas de prevención que pongan énfasis en las serias consecuencias que el abuso sexual tiene para las víctimas.

Este estudio tiene limitaciones ya que los datos fueron obtenidos desde las fichas clínicas, y como el motivo de consulta de la adolescente no era el abuso sexual no fue posible obtener más detalles de la agresión. La población estudiada corresponde al sector socioeconómico medio y bajo, por lo que no es posible generalizar los resultados a la población adolescente de otros estratos socioeconómicos. Sin embargo, si bien este tipo de delito es transversal a la sociedad, mayormente se comete en sectores urbanos de escasos recursos que generan condiciones y prácticas culturales propias, y a la vez, tienden a denunciar más que las víctimas de sectores urbanos de mayores ingresos y mejor nivel educacional.

\section{CONCLUSIÓN}

El antecedente de abuso sexual debe ser considerado en la atención de adolescentes que 
Tabla V

VARIABLES RELACIONADAS CON LA AGRESIÓN POR TIPO DE AGRESIÓN SEXUAL

\begin{tabular}{|c|c|c|c|c|c|}
\hline \multirow{3}{*}{ Variable } & \multicolumn{4}{|c|}{ Tipo de agresión sexual } & \multirow[t]{3}{*}{ Valor $p$} \\
\hline & \multicolumn{2}{|c|}{ Violación } & \multicolumn{2}{|c|}{ Abuso sexual } & \\
\hline & $\mathrm{n}$ & $\%$ & $\mathrm{n}$ & $\%$ & \\
\hline \multicolumn{6}{|c|}{ Revelación agresión } \\
\hline Sí & 44 & 67,7 & 319 & 70,3 & \\
\hline No & 21 & 32,3 & 135 & 29,7 & 0,78 \\
\hline \multicolumn{6}{|c|}{ Duración de la agresión } \\
\hline Reiterado & 26 & 42,6 & 152 & 38,0 & \\
\hline Único & 35 & 57,4 & 248 & 62,0 & 0,58 \\
\hline \multicolumn{6}{|l|}{ Agresor } \\
\hline Familiar & 30 & 42,9 & 255 & 55,3 & 0,07 \\
\hline No familiar & 40 & 57,1 & 206 & 44,7 & \\
\hline \multicolumn{6}{|l|}{ Lugar de agresión } \\
\hline Casa familia & 9 & 31,0 & 27 & 13,2 & 0,03 \\
\hline Casa víctima & 12 & 41,4 & 90 & 43,9 & \\
\hline Otro lugar & 8 & 27,6 & 88 & 42,9 & \\
\hline \multicolumn{6}{|l|}{ A quien reveló } \\
\hline Madre & 23 & 56,1 & 186 & 58,3 & \\
\hline Familiar & 8 & 19,5 & 64 & 20,1 & 0,92 \\
\hline No familiar & 10 & 24,4 & 69 & 21,6 & \\
\hline \multicolumn{6}{|c|}{ Actividad sexual voluntaria } \\
\hline $\mathrm{Si}$ & 55 & 78,6 & 389 & 84,0 & \\
\hline No & 15 & 21,4 & 74 & 16,0 & 0,33 \\
\hline \multicolumn{6}{|l|}{ Parejas sexuales } \\
\hline 1 & 27 & 51,9 & 260 & 67,2 & \\
\hline 2 & 13 & 25,0 & 86 & 22,2 & \\
\hline $3-9$ & 12 & 23,1 & 41 & 10,6 & 0,02 \\
\hline \multicolumn{6}{|l|}{ Consumo de droga } \\
\hline $\mathrm{Si}$ & 35 & 49,3 & 199 & 43,1 & \\
\hline No & 36 & 50,7 & 263 & 56,9 & 0,39 \\
\hline \multicolumn{6}{|c|}{ Consumo de alcohol } \\
\hline $\mathrm{Si}$ & 54 & 76,1 & 372 & 80,0 & \\
\hline No & 17 & 23,9 & 93 & 20,0 & 0,54 \\
\hline \multicolumn{6}{|c|}{ Edad a la entrevista } \\
\hline (promedio, años) & 16,1 & & 15,8 & & 0,02 \\
\hline \multicolumn{6}{|l|}{ Edad a la agresión } \\
\hline (promedio, años) & 10,7 & & 10,1 & & 0,21 \\
\hline \multicolumn{6}{|l|}{ Edad del agresor } \\
\hline (promedio, años) & 23,6 & & 31,9 & & 0,00 \\
\hline \multicolumn{6}{|c|}{ Edad inicio actividad sexual } \\
\hline (promedio, años) & 15,0 & & 14,9 & & 0,62 \\
\hline \multicolumn{6}{|c|}{ Años transcurridos desde la agresión } \\
\hline (promedio, años) & 5,2 & & 5,7 & & 0,30 \\
\hline
\end{tabular}


Tabla VI

VARIABLES RELACIONADAS CON LA AGRESIÓN SEGÚN REVELACIÓN

\begin{tabular}{|c|c|c|c|c|c|}
\hline \multirow{3}{*}{ Variable } & \multicolumn{4}{|c|}{ Revelación de la agresión } & \multirow{3}{*}{ Valor $\mathrm{p}$} \\
\hline & & & & & \\
\hline & $\mathrm{n}$ & $\%$ & $\mathrm{n}$ & $\%$ & \\
\hline \multicolumn{6}{|c|}{ Actividad sexual voluntaria } \\
\hline Sí & 314 & 83,9 & 128 & 81,5 & \multirow[t]{2}{*}{0,58} \\
\hline No & 60 & 16,1 & 29 & 18,5 & \\
\hline \multicolumn{6}{|l|}{ Parejas sexuales } \\
\hline 1 & 208 & 66,9 & 80 & 63,0 & \multirow{3}{*}{0,49} \\
\hline 2 & 69 & 22,2 & 28 & 22,0 & \\
\hline 3 a 9 & 34 & 10,9 & 19 & 15,0 & \\
\hline \multicolumn{6}{|l|}{ Consumo de droga } \\
\hline 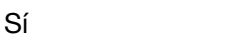 & 152 & 40,7 & 81 & 51,3 & \multirow[b]{2}{*}{0,03} \\
\hline No & 221 & 59,3 & 77 & 48,7 & \\
\hline \multicolumn{6}{|c|}{ Consumo de alcohol } \\
\hline Sí & 295 & 78,5 & 134 & 84,8 & \multirow[t]{2}{*}{0,12} \\
\hline No & 81 & 21,5 & 24 & 15,2 & \\
\hline \multicolumn{6}{|c|}{ Relación víctima-victimario } \\
\hline Familiar & 219 & $\begin{array}{r}76,0 \\
62\end{array}$ & 69 & 23,8 & \multirow[t]{2}{*}{0,00} \\
\hline No familiar & 155 & 63,3 & 90 & 36,7 & \\
\hline \multicolumn{6}{|l|}{ Edad a la entrevista } \\
\hline (promedio, años) & 15,9 & & 15,8 & & 0,86 \\
\hline \multicolumn{6}{|l|}{ Edad a la agresión } \\
\hline (promedio, años) & 10,1 & & 10,2 & & 0,68 \\
\hline \multicolumn{6}{|l|}{ Edad del agresor } \\
\hline (promedio, años) & 31,8 & & 27,4 & & 0,00 \\
\hline \multicolumn{6}{|c|}{ Edad inicio actividad sexual } \\
\hline (promedio, años) & 15,0 & & 14,9 & & 0,68 \\
\hline \multicolumn{6}{|c|}{ Años transcurridos desde agresión } \\
\hline (promedio, años) & 5,7 & & 5,5 & & 0,47 \\
\hline
\end{tabular}

consultan por salud sexual y reproductiva y el equipo de salud debe estar preparado para atender sus múltiples necesidades.

\section{REFERENCIAS}

1. López F, Carpintero E, Hernández A, Martin MJ, Fuertes $A$. Prevalence and sequelae of childhood sexual abuse in Spain. Child Abuse Negl 1995;19:1039-50.

2. Browne A, Finkelhor D. Impact of child sexual abuse: $A$ review of the research. Psychological Bulletin 1986;99:66-77.

3. Cahill C, Llewelyn SP, Pearson C. Long-term effects of sexual abuse which occurred in childhood: A review. Br J Clin Psychol 1991;30:117-30.

4. Finkelhor D, Browne A. The traumatic impact of child sexual abuse: A conceptualization. Am J Orthopsychiatry 1985;55:530-41.

5. Unidad de Estadística y Archivo Medico Legal. 2010.

6. Fergusson DM, Lynskey MT. Childhood sexual abuse and psychiatric disorder in young adulthood I. Prevalence of sexual abuse and factors associated with sexual abuse. J Am Acad Adolesc Psychiatry 1996;34:10-20.

7. Massie M, Johnson S. The importance of recognizing a history of sexual abuse. J Adolesc Health Care 1989;10:184-91.

8. Consentino C, Meyer-Bahlburg H, Albert JL, Weinberg SL. Sexual behavior problems and psychopathology symptoms in sexually abused girls. J Am Acad Child Adolesc Psychiatry 1995;43:1033-42.

9. González-Fortaleza C, Ramos-Lira L, Vignau-Brambila L, Ramírez-Villareal C. El abuso sexual y el intento 
suicida asociados con el malestar depresivo y la ideación suicida de los adolescentes. Salud Mental 2001;24:16-25.

10. Courtois CA. The incest experience and its aftermaths. Victimology 1979;4(4):337-47.

11. Echeburua E, De Corral P. Secuelas emocionales en víctimas de abuso sexual en la infancia. Cuad Med Forense 2006;12:75-82.

12. Beitchman JH, Zucker K, Hood JE, daCosta GA, Akman $D$, Cassavia $E$. A review of the long-term effects of child sexual Abuse. Child Abuse Negl 1992;16:101-18.

13. Mannarino AP, Cohen JA, Smith JA, Moore-Motily S. Six and twelve month follow-up of sexually abused girls. J Interpersonal Violence 1991;6:494-511.

14. Fajewonyomi BA, Orji EO, Adeyemo AO. Sexual dysfunction among female patients of reproductive age in a hospital setting in Nigeria. J Health Popul Nutr 2007;25:10-6.

15. Williams C, Larsen U, McCloskey LA. The impact of childhood sexual abuse and intimate partner violence on sexually transmitted infections. Violence Vict 2010; 25:787-98.

16. Pereda N, Guilera G, Forns M, Gomez-Benito J. The international epidemiology of child sexual abuse: A continuation of Finkelhor 1994. Child Abuse Negl 2009;33:331-42.

17. Buzi RS, Tortolero SR, Roberts RE, Ross MW, Addy RC, Markham CM. The impact of a history of sexual abuse on high-risk sexual behaviors among females attending alternative schools. Adolescence 2003;38:595-605.

18. Finkelhor D, Hotaling G Lewis I, Smith C. Sexual abuse and its relationship to later sexual satisfaction, marital status, religion and attitudes. J Interpersonal Violence 1993;4:279-99.

19. Leserman J. Sexual abuse history: prevalence, health effects, mediators, and psychological treatment. Psychosomatic Med 2005;67:906-15.

20. Chen LP, Murad MH, Paras ML, Colbenson KM. Sexual abuse and lifetime diagnosis of psychiatric disorders: Systematic review and meta-analysis. 2010;85:618-29.

21. Kendall-Tackett AK, Meyer Williams L, Finkelhor D. Impact of sexual abuse on children: A review and synthesis of recent empirical studies. Psyhological Bulletin 1993;113:164-80.

22. Brown NL, Wilson SR, Kao YM, Luna V, Kuo ES, Rodríguez C, Lavori PW. Correlates of sexual abuse and subsequent risk taking. Hispanic J Behavioral Sciences 2003; 25:331-51.

23. Ramos-Lira L, Saldívar-Hernández G, Medina-Mora M E, Rojas-Guiot E, Villatoro-Velásquez J. Prevalencia de abuso sexual en estudiantes y su relación con el consumo de drogas. Salud Pública de México 1998;40(3):221-33.

24. Klein H, Chao BS. Sexual abuse during childhood and adolescence as predictors of HIV-related sexual risk during adulthood among female sexual partners of injection drug users. Violence Against Women 1995;1:55-76.

25. Vitriol GV, Vásquez M, Iturra LI, Muñoz RC. Diagnóstico y abordaje de secuelas por abuso sexual infantil, en tres mujeres consultantes a un servicio de salud mental de hospital general", Rev Chil Neuro Psiquiat 2007;45:20-8.

26. Twill SE, Green DM, Traylor Amy. A descriptive study on sexually exploited children in residential treatment. Child Youth Care Forum 2010; 39:187-199.

27. Ramos-Lira L, Saltijeral-Méndez MT, Romero MM, Caballero GMA, Martínez-Vélez NA. Violencia sexual y problemas asociados en una muestra usuarias de un centro de salud. Salud Pública de México 2001;43:182-91.

28. Pou Fernández J, Ruiz España A, Comas Masmitjá LI. Abuso sexual. Experiencia en una unidad funcional de abusos a menores. Anales Españoles de Pediatría 2001;54:243-50.

29. Guerra O. Abuso sexual en niñas y niños. Consideraciones clínicas. Rev Chil Pediatr 2000;71:368-75.

30. Molina R, González E, Sandoval J. Embarazo en adolescentes producto de una agresión sexual: alto riesgo obstétrico y perinatal, Rev Soc Chil Obst Gin Infantil Adolescencia 1995;2(3):74-82.

31. González E, Molina T, Toledo V, Luengo X, Molina C. Violación intra y extrafamiliar en adolescentes: Variables personales y familiares seleccionadas. Rev Soc Chil Obst Gin Infantil Adolescencia 1997;4:13-21.

32. Warlick AC, Mathews R, Gerson CA. Keeping childhood sexual abuse on the urologic radar screen. Urology 2005;66:1143-9.

33. Senn ET, Carey PM, Vanable AP. Childhood and adolescent sexual abuse and subsequent sexual risk behavior: evidence from controlled studies, methodological critique, and suggestions for research. Clin Psichol Rev 2008;28:711-35.

34. Código Penal de Chile. Ley 19.617 y Ley 19.927. 